\title{
Encoding of Gustatory Working Memory by Orbitofrontal Neurons
}

\author{
Antonio H. Lara, Steven W. Kennerley, and Jonathan D. Wallis \\ Department of Psychology and Helen Wills Neuroscience Institute, University of California at Berkeley, Berkeley, California 94720-3190
}

The content model regarding the functional organization of working memory in prefrontal cortex (PFC) states that different PFC areas encode different types of information in working memory depending on their afferent connections with other brain areas. Previous studies that tested this model focused on visual, auditory and somatosensory information. However, posterior areas processing this information project to widespread and overlapping regions of lateral PFC, making it difficult to establish the veracity of the model. In contrast, gustatory information enters PFC via orbitofrontal cortex (OFC), and so the content model would argue that OFC should be responsible for maintaining gustatory information in working memory. To test this, we recorded the activity of single neurons throughout PFC and gustatory cortex (GUS) from two subjects while they performed a gustatory delayed-match-to-sample task with intervening gustatory distraction. Neurons that encoded the identity of the gustatory stimulus across the delay, consistent with a role in gustatory working memory, were most prevalent in OFC and GUS compared with dorsolateral PFC and ventrolateral PFC. Gustatory information in OFC was more resilient to intervening distraction, paralleling previous findings regarding visual working memory processes in PFC and posterior sensory cortex. Our findings provide support for the content model of working memory organization. Maintaining gustatory information may be one aspect of a wider function for OFC in reward working memory that could contribute to its role in decision-making.

Key words: prefrontal cortex; working memory; gustatory; executive; frontal; reward

\section{Introduction}

The prefrontal cortex (PFC) is responsible for working memory, but the precise organization of the neuronal mechanisms underlying this cognitive function remain unclear. One prominent model emphasizes an organization based on the content of the sensory information that is to be maintained in working memory (Goldman-Rakic, 1987, 1996). Different subregions of PFC receive inputs related to the processing of specific types of information and are subsequently responsible for maintaining and processing that information in working memory. Support for this model came from studies showing that lesions of dorsolateral PFC (DLPFC), which strongly connects with parietal cortex (Cavada and Goldman-Rakic, 1989), impaired spatial working memory (Funahashi et al., 1993), whereas lesions of ventrolateral PFC (VLPFC), which strongly connects with inferior temporal cortex, impaired object working memory (Mishkin and Manning, 1978). Early neurophysiological studies also supported this distinction: neurons maintaining spatial information in working memory

\footnotetext{
Received Sept. 26, 2008; revised Dec. 5, 2008; accepted Dec. 7, 2008.

This work was supported by National Institute on Drug Abuse Grant R01DA19028 and National Institute of Neurological Disorders and Stroke Grant P01NS040813 to J.D.W., and National Institute of Mental Health Training Grant F32MH081521 to S.W.K. A.H.L. contributed to all aspects of the project, S.W.K. contributed to experimental design and data collection, and J.D.W. contributed to experimental design, data analysis, writing of this manuscript and supervision of the project.

The authors declare no competing financial interests.

Correspondence should be addressed to Jonathan D. Wallis, 132 Barker Hall, University of California at Berkeley,

Berkeley, CA 94720-3190. E-mail: wallis@berkeley.edu.

D0I:10.1523/JNEUROSCI.4637-08.2009

Copyright $\odot 2009$ Society for Neuroscience $\quad 0270-6474 / 09 / 290765-10 \$ 15.00 / 0$
}

occurred in DLPFC (Funahashi et al., 1989), whereas those maintaining object information were in VLPFC (Wilson et al., 1993).

Recent findings argue against the content model. For example, when monkeys have to remember both the identity and spatial location of an object, neurons encoding both attributes occur throughout lateral PFC, including both DLPFC and VLPFC subregions (Rao et al., 1997). Furthermore, PFC neurons can switch whether they encode information about an object's identity or location depending on which aspect is currently relevant to the task (Rao et al., 1997). These findings suggest that PFC organization does not depend on the nature of the information encoded by the neurons. However, evidence has also increased for integration of spatial and object information in posterior sensory cortex. For example, neurons in parietal cortex, traditionally associated with encoding spatial information, will encode the color of objects if color is behaviorally relevant (Toth and Assad, 2002). If integration of spatial and object information occurs in posterior sensory cortex, it is not surprising that the activity of PFC neurons reflects this integration. Consideration of other sensory modalities might provide us with a stronger test of the content model.

Researchers have also studied working memory for auditory and somatosensory information at the single neuron level (Romo et al., 1999; Romanski and Goldman-Rakic, 2002). However, information from these modalities predominantly enters the frontal lobe via VLPFC (Carmichael and Price, 1995; Romanski et al., 1999) and so consequently they do not provide a stronger test of the content model than has already been established by studying 
the visual modality. In contrast, orbitofrontal cortex (OFC) is the major recipient in the frontal lobe of both gustatory and olfactory information (Morecraft et al., 1992; Barbas, 1993). Thus, the content model would predict that OFC but not VLPFC neurons would encode olfactory and gustatory information in working memory. To test this, we simultaneously recorded neuronal activity from multiple PFC subregions (DLPFC, VLPFC and OFC) and primary gustatory cortex (GUS), while subjects performed a gustatory delayed match-to-sample (DMS) task.

\section{Materials and Methods}

Subjects and neurophysiological procedures. The subjects were two male rhesus monkeys (Macaca mulatta). Subject $\mathrm{C}$ was 5 years old at the time of recording and weighed $13 \mathrm{~kg}$. Subject $\mathrm{G}$ was 4 years old and weighed 9 $\mathrm{kg}$. We regulated their daily fluid intake to maintain motivation on the task. Our methods for neurophysiological recording are reported in detail previously (Wallis and Miller, 2003). Briefly, we implanted both subjects with a head positioner for restraint and two recording chambers, one over each hemisphere. The first chamber allowed access to PFC, and the second chamber allowed access to GUS. In subject C, we recorded from PFC in the right hemisphere and GUS in the left hemisphere. In subject G, we recorded from GUS in the right hemisphere and PFC in the left hemisphere.

Our recordings from DLPFC were dorsal to the principal sulcus consisting of areas 9 and 9/46. Our recordings from VLPFC were ventral to the principal sulcus consisting of areas $47 / 12$ and 45 . Our recordings from OFC were located between the lateral and medial orbital sulci. We focused on area 13 , because this is a region in which taste responsive neurons are particularly found (Pritchard et al., 2005), but our recordings also included some neurons in area 11 . We also recorded from GUS, so that we could compare neuronal activity in PFC with neuronal activity in the primary sensory cortex responsible for the detection and encoding of juice in the mouth. Unlike GUS, OFC does not have direct connections with the thalamic taste area (Pritchard et al., 1986): gustatory information can only reach OFC via GUS. Thus, we could compare and contrast neuronal activity in OFC with the upstream area from which it is receiving gustatory information.

We determined the positions of the recording chambers using a $1.5 \mathrm{~T}$ magnetic resonance imaging (MRI) scanner. We recorded simultaneously from the different brain areas using arrays of 8-16 tungsten microelectrodes (FHC Instruments). We determined the approximate distance to lower the electrodes from the MRI images and advanced the electrodes using custom-built, manual microdrives until they were located just above the cell layer. We then slowly lowered the electrodes into the cell layer until we obtained a neuronal waveform. We randomly sampled neurons; no attempt was made to select neurons based on responsiveness. This procedure ensured an unbiased estimate of neuronal activity thereby allowing a fair comparison of neuronal properties between the different brain regions. Waveforms were digitized and analyzed off-line (Plexon Instruments). All procedures were in accord with the National Institutes of Health guidelines and the recommendations of the University of California, Berkeley Animal Care and Use Committee.

We reconstructed our recording locations by measuring the position of the recording chambers using stereotactic methods. We plotted the positions onto the MRI sections using commercial graphics software (Adobe Illustrator). We confirmed the correspondence between the MRI sections and our recording chambers by mapping the position of sulci and gray and white matter boundaries using neurophysiological recordings. We traced and measured the distance of each recording location along the cortical surface from the lip of the ventral bank of the principal sulcus. We also measured the positions of the other sulci, relative to the principal sulcus, allowing the construction of the unfolded cortical maps (see Fig. 2).

Behavioral task. The subject sat in a primate chair in front of a computer screen. Two computers running NIMH Cortex were used to control the task. To start the task the subject held a lever for $1000 \mathrm{~ms}$. A picture appeared on the screen for $650 \mathrm{~ms}$ followed by $500 \mathrm{~ms}$ of juice delivery to the subject's mouth (Fig. 1). The picture helped warn the

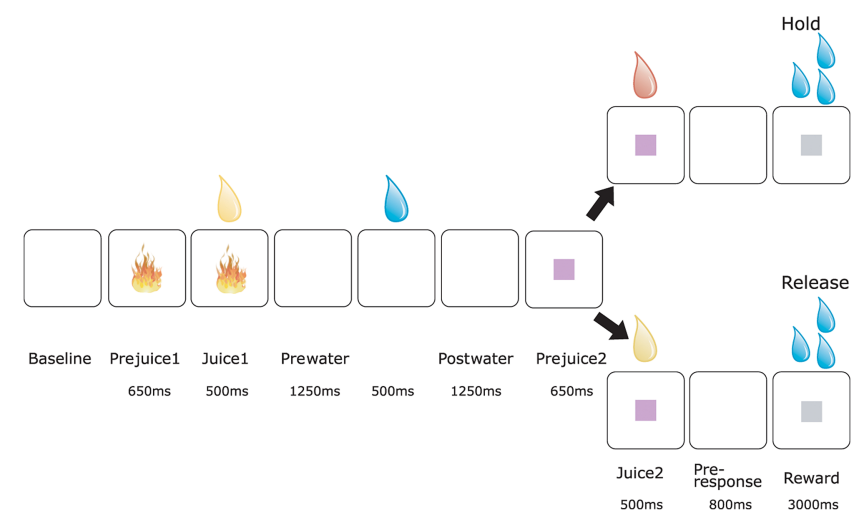

Figure 1. Illustration of the behavioral task. The subject grasped a lever to initiate the trial. We presented two juices sequentially separated by a brief delay. The subject had to release a lever if the two juices were the same (match) or continue holding the lever if the two juices were different (nonmatch). We presented a water drop half way through the delay which served as a distractor and ensured that remnants of the first juice were rinsed from the subject's mouth.

subject of the juice's delivery and enabled us to examine neuronal selectivity relating to the expectancy of receiving a specific juice. However, the subject could not use it to solve the task (see Results). The picture remained on the screen until the end of the juice delivery. We used three different juice stimuli: each of the juices was predicted by one of two pictures (requiring six pictures total) thereby enabling us to distinguish neuronal responses related to the expectancy of a specific juice from neuronal responses related to encoding the visual properties of the picture. The juices we used were orange juice (Berkeley Farms), vegetable juice (V8; CSC Brands) and $0.1 \mathrm{~m}$ sucrose solution mixed with guava flavoring (LorAnn Oils). We delivered them using a peristaltic pump (ISMATEC), at a constant flow rate of $0.4 \mathrm{ml} \mathrm{s}^{-1}$, through a bundle of stainless steel cannulae (13 gauge, Small Parts). We prevented crosscontamination of juice flavors by delivering each juice down a specific cannula.

After a $1250 \mathrm{~ms}$ delay, we delivered $500 \mathrm{~ms}$ of water. This served as a distractor and helped ensure that the subject's mouth was rinsed of any remnants of the first juice. After a second $1250 \mathrm{~ms}$ delay, a lilac square appeared at fixation for $650 \mathrm{~ms}$. It was followed by $500 \mathrm{~ms}$ of juice delivery. On one-half of the trials, the second juice was the same as the first juice (match), whereas on the other half of the trials it was one of the two other juices (nonmatch). After a delay of $800 \mathrm{~ms}$, a gray square appeared and the subject had to indicate whether the two juices were the same or different by either immediately releasing the lever (match) or holding (nonmatch) the lever for $900 \mathrm{~ms}$. Thus, to solve the task the subject must hold the first juice stimulus in working memory for the 3000 $\mathrm{ms}$ delay between juices, while ignoring the intervening water drop. We chose to deliver the water drop in the middle of the $3000 \mathrm{~ms}$ delay so that we would have two $1250 \mathrm{~ms}$ epochs, before and after the water drop, in which we could compare neuronal selectivity and determine unambiguously the effect of the water drop on ongoing neuronal activity. At the end of the trial, we rewarded correct responses with $2000 \mathrm{~ms}$ of water. Incorrect responses resulted in the screen turning red for 10-s and the subject received no reward. There was a $3000 \mathrm{~ms}$ intertrial interval (ITI).

To determine the neuronal response purely to juice stimuli, on one third of the trials the subject received a $500 \mathrm{~ms}$ juice stimulus outside of the context of the task. We term these "free-juice" trials. The subject took hold of the lever but after $1000 \mathrm{~ms}$, rather than a picture appearing, a juice drop was delivered. These trials were randomly interspersed with task trials throughout the session. All three juice stimuli were given and the subjects could not predict when these trials would occur.

Statistical methods. We only analyzed correct trials. There were too few error trials to permit meaningful analysis of neuronal activity on those trials. We constructed spike density histograms by averaging activity across the appropriate conditions using a sliding window of $100 \mathrm{~ms}$.

We quantified neuronal selectivity during the gustatory DMS task using several defined time epochs. To analyze selectivity relating to the 
expectancy of receiving a specific juice reward, we defined the prejuice 1 epoch beginning $100 \mathrm{~ms}$ after the first picture appears on the screen and lasting $550 \mathrm{~ms}$ until the delivery of the first juice (we discarded the first $100 \mathrm{~ms}$ to account for the latency of neuronal responses to visual stimuli in PFC). To analyze selectivity relating to the initial identification of the juice, we defined the juicel epoch as beginning $250 \mathrm{~ms}$ after onset of the first juice and lasting $500 \mathrm{~ms}$ until $250 \mathrm{~ms}$ after the first juice offset. The $250 \mathrm{~ms}$ offset allowed for the latency of gustatory responses as determined from the free-juice trials (see Results). To analyze selectivity relating to gustatory working memory, we defined two epochs either side of the distracting water drop. The prewater epoch starts $250 \mathrm{~ms}$ after the offset of the first juice and lasts $1000 \mathrm{~ms}$ until the delivery of the water drop. The postwater epoch starts $250 \mathrm{~ms}$ after the offset of the water drop and lasts $1000 \mathrm{~ms}$ until the appearance of the second picture on the screen.

For each neuron and each epoch in turn, we calculated the neuron's mean firing rate. We performed a two-way ANOVA on the firing rate, using factors of juice (the identity of the first juice) and set (the picture set from which we drew the reward-predictive cue), evaluating significance using an $\alpha$ level of $p<0.01$. We then used $\chi^{2}$ tests to determine whether there were significant differences in the prevalence of neurons encoding the different experimental factors in different brain areas. We defined juice-selective neurons as those that showed a significant main effect of juice with no other main effects or interactions. We also used the twoway ANOVA to calculate the strength of selectivity. We did this by calculating the magnitude of our statistical effects using $\eta$-squared. This is equivalent to the percentage of explained variance attributable to the juice manipulation $\left(\mathrm{PEV}_{\text {juice }}\right)$. It is calculated by dividing the sum of squares associated with the juice manipulation by the total sum of squares in the analysis and multiplying the result by $100 \%$.

To analyze the free-juice trials, we defined two time epochs. The juice epoch consisted of the $500 \mathrm{~ms}$ of juice delivery. The postjuice epoch started $250 \mathrm{~ms}$ after the offset of the juice and lasted $1000 \mathrm{~ms}$. This epoch was designed to be comparable to the prewater epoch in the gustatory DMS task. For each neuron and each epoch, we calculated the neuron's mean firing rate. To determine the prevalence of neurons encoding the juice, we performed a one-way ANOVA on the firing rate, using the factor of the juice's identity. We also used the free-juice trials to determine the latency at which neurons encoded the identity of the juice by performing a "sliding" ANOVA analysis. We took a $200 \mathrm{~ms}$ window of time, beginning $500 \mathrm{~ms}$ before the onset of juice, and performed a oneway ANOVA on the firing rate, using the factor of the juice's identity. We then moved the window forward by $10 \mathrm{~ms}$, and repeated the analysis. We continued in this manner until we had analyzed up to $500 \mathrm{~ms}$ after the offset of the juice. We defined the latency of selectivity as the time when the $p$-value fell below 0.005 for three consecutive time bins. We chose this criterion by comparing it with the selectivity evident in the spike density histograms. However, to verify that it was indeed a sensible criterion, we examined how many neurons would have reached the criterion in the $1000 \mathrm{~ms}$ before the delivery of the juice (i.e., when it would have been impossible for the neurons to encode the juice's identity). Just 19 of 392 $(4.8 \%)$ neurons reached criterion in this time period, indicating that our choice of criterion yielded a reasonable type I error.

To analyze the processes that underlie the subject's decision as to whether or not the two presented juices match, we focused on the period of the trial that followed the presentation of the second juice. We defined two time epochs. The juice 2 epoch began $250 \mathrm{~ms}$ after the onset of the second juice delivery and lasted $500 \mathrm{~ms}$ until $250 \mathrm{~ms}$ after the offset of the juice. The preresponse epoch began $250 \mathrm{~ms}$ after the offset of the juice and lasted $650 \mathrm{~ms}$ until $100 \mathrm{~ms}$ after the onset of the go cue. For each neuron and each epoch, we calculated the neuron's mean firing rate. We performed a two-way ANOVA on the firing rate, using factors of juice (the identity of the second juice) and response (whether the subject would indicate match or nonmatch). We calculated the magnitude of our statistical effects using $\eta$-squared.

To calculate the latency at which the neurons determined the behavioral response, we performed a sliding two-way ANOVA using factors of juice (the identity of the second juice) and response (whether the subject would indicate match or nonmatch) as the experimental factors. Our methods were analogous to those used to determine the latency for encoding the juice's identity on the free-juice trials. We defined the latency of selectivity as the time when the $p$-value for the main effect of response fell below 0.005 for three consecutive time bins. We verified that this was a sensible criterion by calculating that just 19 of $392(4.8 \%)$ neurons reached this criterion in the $1000 \mathrm{~ms}$ before the delivery of the second juice (i.e., when it would have been impossible for the subject to know the correct behavioral response).

Finally, we compared each neuron's overall firing rate in each task epoch to its firing rate during a baseline epoch that consisted of the 1000 $\mathrm{ms}$ between the subject first grasping the lever to initiate the trial and the presentation of the first picture. We compared these firing rates using a $t$ test with an $\alpha$ level of 0.01 . This analysis enabled us to detect those neurons that were not encoding gustatory information per se, but were modulating their firing rate in response to task-related events.

\section{Results}

\section{Behavior}

Both monkeys performed the task well above the chance level of $50 \%$ (C $82 \pm 5.7 \%$ over 40 recording sessions, G $84 \pm 5.1 \%$ correct over 19 recording sessions). Subject C completed a mean of 377 correct trials ( \pm 91 trials) per session and subject $\mathrm{G}$ completed 419 correct trials ( \pm 41 trials) per session. Subject C showed significantly better performance when he had to remember guava $(86 \pm 7.7 \%)$ or tomato $(85 \pm 8.2 \%)$ compared with orange $\left(76 \pm 9.4 \%\right.$, one-way ANOVA, $F_{(2,117)}=18, p<5 \times$ $\left.10^{-7}\right)$. Subject $\mathrm{G}$ showed no differences in performance between the three juices (orange: $81 \pm 10 \%$, guava: $84 \pm 7.4 \%$, tomato: $87 \pm 5.0 \%$; one-way ANOVA, $\left.F_{(2,51)}=2.1, p>0.1\right)$. Both subjects performed better on nonmatch conditions compared with match (C $91 \pm 4.5 \%$ nonmatch, $74 \pm 12 \%$ match, one-way ANOVA, $F_{(1,78)}=68, p<5 \times 10^{-12} ; \mathrm{G} 89 \pm 4.2 \%$ nonmatch $79 \pm 11 \%$ match, one-way ANOVA, $\left.F_{(1,34)}=13, p<0.005\right)$. Subject $C$ had a median reaction time of $336 \pm 66 \mathrm{~ms}$, whereas $\mathrm{G}$ had a median reaction time of $251 \pm 27 \mathrm{~ms}$.

We also conducted a behavioral probe test to confirm that the subjects were solving the task in the manner we anticipated. We ensured that the subjects were not solving the task by remembering the identity of the reward-predictive pictures rather than the identity of the juice. Such an explanation would be necessarily convoluted, because although they could remember the picture, they would still need to recall by the end of the delay what juice was paired with that picture to determine whether or not the second juice matched the first. Nevertheless, we tested whether the subjects had adopted this strategy by replacing the rewardpredictive pictures with gray squares for one session. This behavioral manipulation had little effect on our subject's performance. Subject C performed at $84 \%$ (compared with $82 \%$ with the pictures), whereas subject $\mathrm{G}$ performed at $76 \%$ (compared with $84 \%$ with the pictures). Thus, the subjects did not appear to be using the reward-predictive cues to solve the task.

\section{Neurophysiological results}

We recorded neuronal activity simultaneously from four cortical areas, DLPFC, VLPFC, OFC and GUS. We recorded from 392 neurons. There were 141 cells in DLPFC (C 58, G 83), 89 cells in VLPFC (C 40, G 49), 77 cells in OFC (C 46, G 31) and 85 cells in GUS (C 54, G 31). Figure 2 illustrates the locations from which we recorded.

\section{Neuronal responses during the gustatory DMS task}

For each neuron and each of the first four epochs in turn, we used a two-way ANOVA to determine whether the neuron encoded the identity of the juice or the visual properties of the rewardpredictive cue (see Materials and Methods). Very few neurons 


\section{Subject C}

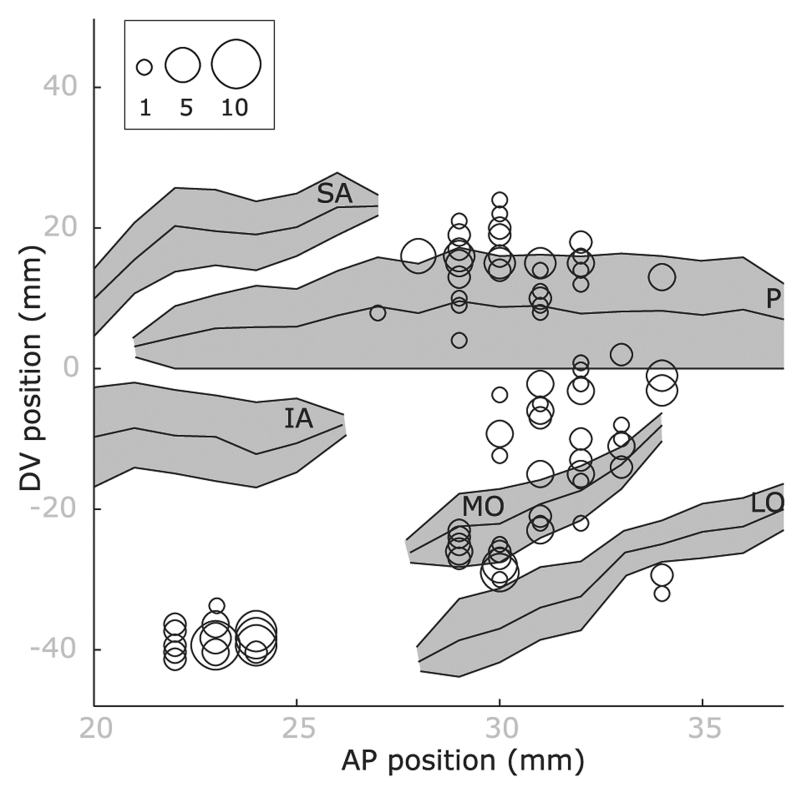

Subject G

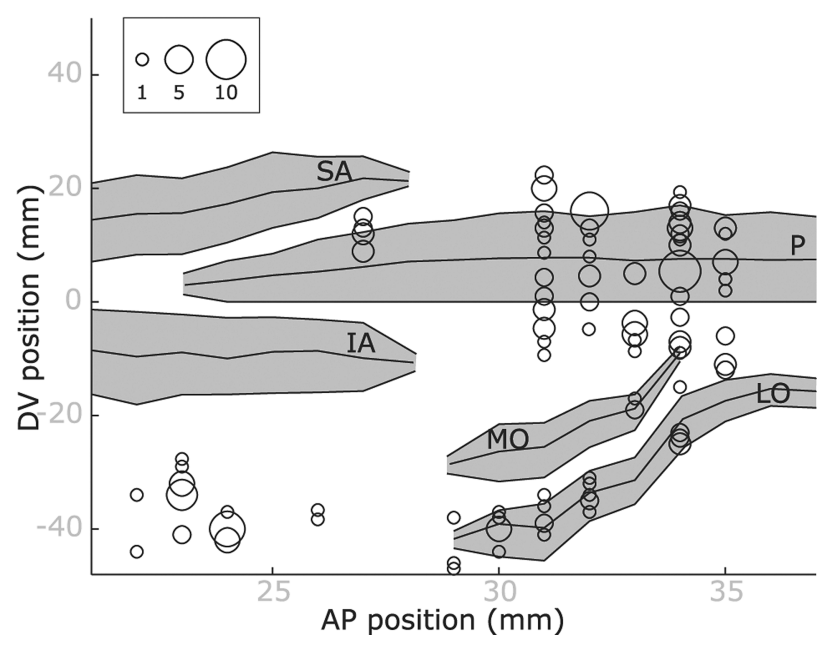

Figure 2. Flattened reconstructions of the cortex indicating the locations of recorded neurons. The size of the circles indicates the number of neurons recorded at that location. We measured the anterior-posterior position from the interaural line ( $x$-axis), and the dorsoventral position relative to the lip of the ventral bank of the principal sulcus ( 0 point on $y$-axis). Gray shading indicates unfolded sulci. See Materials and Methods for details regarding the reconstruction of the recording locations. SA, superior arcuate sulcus; IA, inferior arcuate sulcus; $P$, principal sulcus; LO, lateral orbital sulcus; M0, medial orbital sulcus.

showed evidence of encoding the visual properties of the rewardpredictive cue. Only 21 of 392 (5\%) of the neurons showed a main effect of picture set in at least one of the four epochs. This did not significantly differ from what would have been expected by chance $(0.01 \alpha$ level $\times 4$ epochs $=0.04$, binomial, $p>0.1)$. A similar number of neurons ( 16 of 392 or $4 \%$ ) showed a significant interaction between juice and picture set in at least one of the four epochs. This proportion also did not significantly differ from what would have been expected by chance (binomial, $p>0.1$ ). Consequently, the remainder of our report will focus on those neurons that showed a main effect of juice, but with no significant main effect or interaction with picture set.

Reward expectancy. We found neurons that showed a selective modulation of their firing rate during the prejuicel epoch de- pending on which juice the subject anticipated receiving. Figure $3 A$ shows a neuron that has a significantly higher firing rate before the onset of the first juice only when the juice to be delivered was orange juice (two-way ANOVA, $F_{(2,193)}=15.2, p<1 \times$ $\left.10^{-6}\right)$. Such a neuron is consistent with previous reports detailing the encoding of expected rewards in PFC (Watanabe, 1996; Tremblay and Schultz, 1999; Hikosaka and Watanabe, 2000). This type of encoding was a lot more prevalent in PFC than GUS (Table 1). A statistical comparison of the proportion of selective neurons in the different brain areas confirmed that all the comparisons between PFC and GUS were significant $\left(\chi^{2}>5, p<\right.$ $0.05)$ whereas none of the comparisons between the different PFC areas reached significance $\left(\chi^{2}<1, p>0.1\right)$. Indeed, the proportion of selective neurons in GUS did not exceed that expected by chance (binomial test, $p>0.1$ ).

Juice identification. Many neurons encoded the identity of the first juice. The neuron in Figure $3 B$ shows a significantly higher firing rate during the juicel epoch for guava and tomato juice compared with orange juice (two-way ANOVA, $F_{(2,270)}=14.1$, $\left.p<1 \times 10^{-5}\right)$. Neurons of this type were present in all four cortical areas from which we recorded (Table 1) with no significant differences among areas (all comparisons $\left.\chi^{2}<1.8, p\right\rangle$ 0.05).

Gustatory working memory. Many neurons showed a selective modulation of firing rate during the delay dependent on the identity of the first juice, consistent with a role in gustatory working memory. Figure $3 C$ shows such a neuron recorded from OFC. It had a significantly higher firing rate when the subject was holding orange or tomato juice in working memory compared with guava juice $\left(F_{(2,280)}=150, p<1 \times 10^{-16}\right)$. The difference in firing rate was still evident after the intervening water drop $\left(F_{(2,280)}=85\right.$, $\left.p<1 \times 10^{-16}\right)$. However, not all neurons maintained their selectivity across the entire delay. The GUS neuron in Figure $3 D$ shows a significantly higher firing rate when the subject was holding orange or tomato juice in working memory compared with guava juice during the prewater epoch $\left(F_{(2,222)}=10, p<0.0001\right)$, but the selectivity disappeared after the water drop $\left(F_{(2,222)}<1\right.$, $p>0.1)$. For such a neuron, the activity during the prewater epoch might reflect an ongoing taste response produced by the first juice, which is subsequently eliminated by the delivery of the intervening water drop.

We compared the proportion of juice-selective neurons between the different areas during both delay epochs. Neurons encoding the juice's identity across the delay were common in GUS and OFC, but were relatively infrequent in DLPFC and VLPFC (Table 1). We used $\chi^{2}$ tests to determine whether the difference in the prevalence of these neurons in the different areas was significant. During the prewater epoch there were significantly more selective neurons in GUS compared with DLPFC and VLPFC (both comparisons, $\left.\chi^{2}>9, p<0.01\right)$. However, there was no significant difference between GUS and OFC $\left(\chi^{2}=1.7, p>0.1\right)$. Finally, there were no significant differences between DLPFC, VLPFC and OFC (all comparisons, $\chi^{2}<3.6, p>0.05$ ). During the postwater epoch, there were significantly more neurons in OFC and GUS relative to DLPFC $\left(\chi^{2}>4, p<0.05\right)$. All other comparisons were not significant $\left(\chi^{2}<2.4, p>0.05\right)$.

Next, for each area in turn, we examined whether there was a significant difference in the proportion of selective neurons during the pre- and postwater epochs. For all three PFC areas, there was no significant difference in the prevalence of the juiceselective neurons during the pre- and postwater epochs (all comparisons $\left.\chi^{2}<1.1, p>0.05\right)$. However, in GUS there were significantly fewer selective neurons in the postwater epoch compared 
A
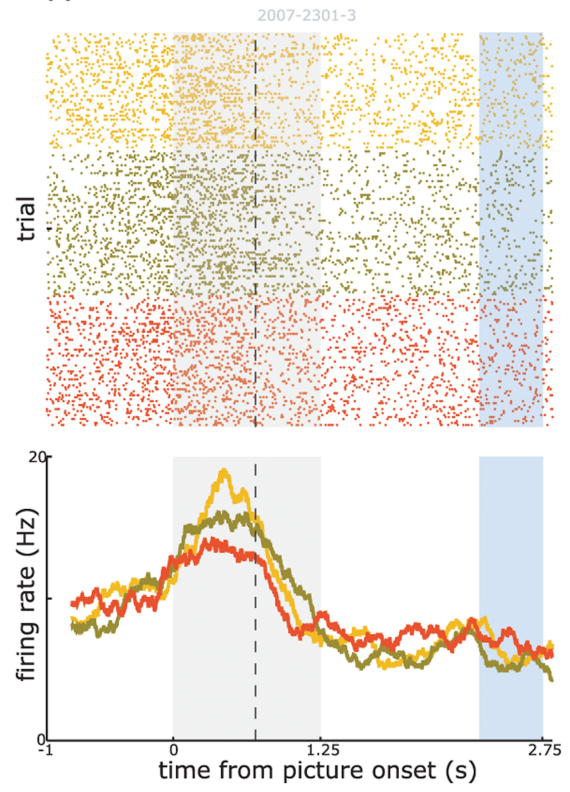

C
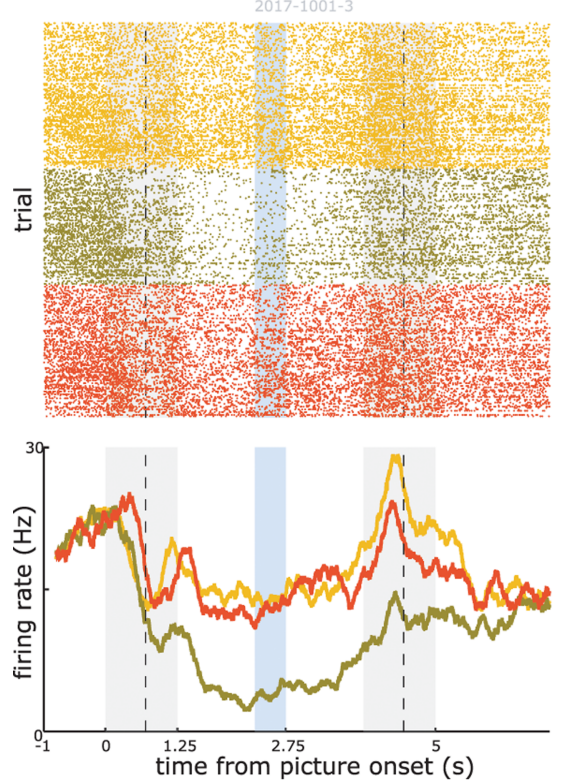

B
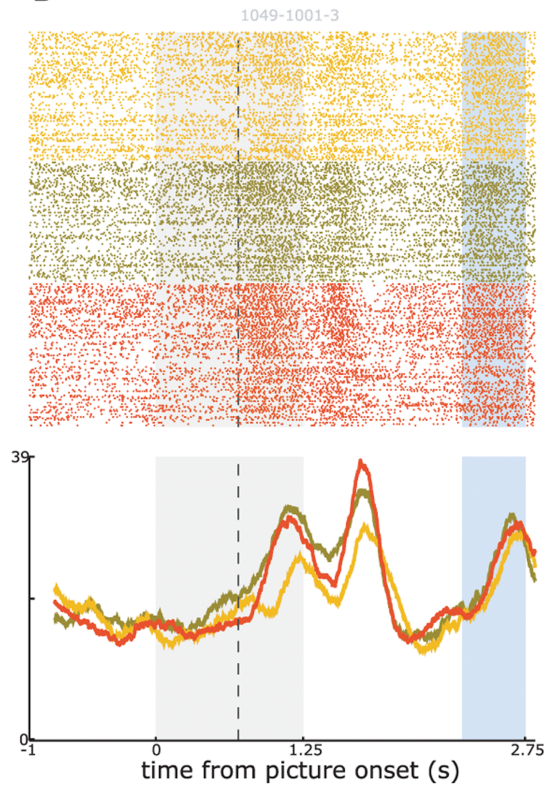

orange

guava

D
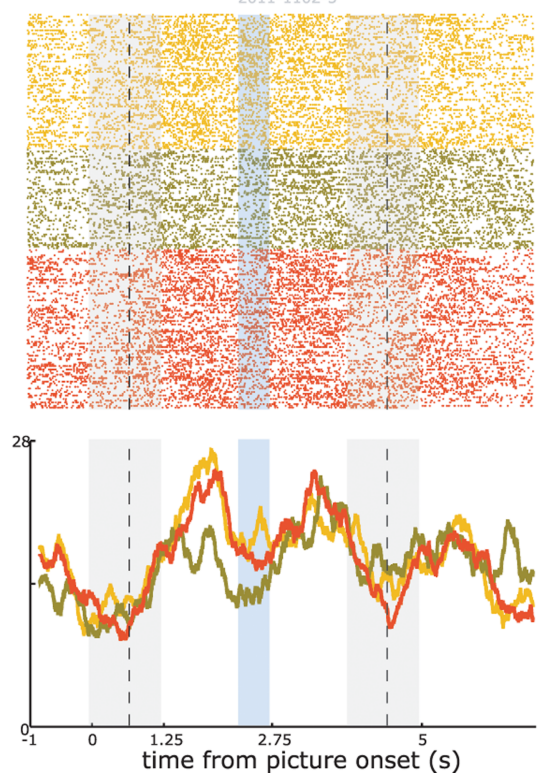

Figure 3. A, Raster plot and spike density histogram illustrating an $\mathrm{OFC}$ neuron that had a significantly higher firing rate when the subject expected to receive orange juice. The gray shading illustrates the prejuice and juice epochs with the vertical dotted line indicating the onset of juice delivery. The blue shading illustrates the delivery of the water drop. $B$, An OFC neuron that had a significantly higher firing rate to the delivery of guava and tomato juice relative to orange juice. C, An OFC neuron that showed a significantly higher firing rate to the delivery of orange juice and tomato juice relative to guava juice. The encoding lasted throughout the prewater epoch and survived the delivery of the water drop. D, A GUS neuron that had a significantly higher firing rate to the delivery of orange juice and tomato juice relative to guava juice that lasted through the prewater epoch. The encoding disappeared after the delivery of the water drop.

with the prewater epoch $\left(\chi^{2}=3.94, p<0.05\right)$. Thus, PFC neurons were better able to maintain information about the juice across the intervening water drop than GUS neurons.

To determine the strength of neuronal selectivity (as opposed to its prevalence), we calculated $\mathrm{PEV}_{\text {juice }}$ for all neurons during the pre- and postwater epochs (see Materials and Methods). Figure 4 shows the mean value of this measure for each of the four brain areas and two time epochs. Neurons in OFC and GUS encoded the juice during the delay more strongly compared with
DLPFC and VLPFC. In addition, OFC neurons encoded the juice in working memory with approximately the same strength in the pre- and postwater epochs whereas GUS neurons showed weaker selectivity during the postwater epoch compared with the prewater epoch. To analyze these effects statistically, we performed a two-way ANOVA with area (the four different brain areas) and epoch (pre- and postwater) as factors. There was a significant area $\times$ epoch interaction $\left(F_{(3,776)}=\right.$ $3.3, p<0.05$ ) which we characterized by analyzing the simple effects and using post hoc Tukey-Kramer tests (evaluated at $p<$ $0.05)$. There was a significant simple effect of area on both the prewater $\left(F_{(3,776)}=14\right.$, $\left.p<5 \times 10^{-9}\right)$ and postwater epochs $\left(F_{(3,776)}=2.9, p<0.05\right)$. Post hoc tests revealed that during the prewater epoch, mean $\mathrm{PEV}_{\text {juice }}$ in GUS was significantly higher than DLPFC and VLPFC and significantly higher in OFC than DLPFC. For the postwater epoch, mean $\mathrm{PEV}_{\text {juice }}$ in OFC was significantly higher compared with both DLPFC and VLPFC but not GUS. The simple effect of epoch on each of the four areas was only significant for GUS $\left(F_{(1,776)}=15, p<0.0005\right.$, all other areas, $p>0.1)$. GUS neurons had significantly weaker selectivity during the postwater epoch compared with the prewater epoch, whereas in the other areas the strength of selectivity remained unchanged after the intervening water distractor.

In summary, many neurons showed activity consistent with encoding of gustatory information in working memory. Based on the prevalence of selective neurons and the overall strength of selectivity, OFC and GUS appeared to be more involved in encoding gustatory information than DLPFC and VLPFC. However, whereas the intervening gustatory distraction had little effect on OFC activity, it significantly weakened the mnemonic trace in GUS. This suggests that the encoding of gustatory information in GUS is more of a sensory response to the taste of the first juice, whereas that in OFC is more consistent with gustatory working memory. OFC neurons continue to encode gustatory information despite the fact that the intervening water drop disrupted encoding in the upstream area from which they receive this information.

Characterization of the neuronal encoding of the juices. There appeared to be two distinct ways of encoding the juices prominent in our neuronal populations. Some neurons, such as the neuron in Figure 3A, showed an increase in firing rate for one of the juices relative to the other two juices. We refer to such encoding as "standard selectivity" because this is the pattern one typically envisages when discussing neuronal selectivity. In contrast, 
other neurons, such as those in Figure $3 B-D$, showed a lower firing rate to one of the juices relative to the other two juices. We refer to such encoding as "inverse selectivity." We determined the relative prevalence of these two types of selectivity in each brain area. For each neuron that showed selectivity in any of the four epochs (as defined by the two-way ANOVA) we determined the epoch in which it showed maximal selectivity (calculated via $\mathrm{PEV}_{\text {juice }}$ ). We then calculated its mean firing rate for each of the three juices in turn as well as its overall mean firing rate during this epoch. We determined the number of neurons for which the mean firing rate of just one of the juices exceeded the overall mean firing rate (consistent with standard selectivity), and the number of neurons for which the mean firing rate of two of the juices exceeded the overall mean firing rate (consistent with inverse selectivity). In DLPFC and OFC, approximately half the neurons showed the standard pattern of selectivity whereas the remainder showed the inverse pattern (DLPFC, 18 of 36 or $50 \%$; OFC, 15 of 29 or $52 \%$; binomial test, $p>0.1$ in both cases). In VLPFC and GUS, the standard pattern of selectivity dominated (VLPFC, 23 of 31 or $74 \%$; GUS, 25 of 36 or $69 \%$; binomial test, $p<0.05$ in both cases).

We also examined whether there was any consistency in the rank order of mean firing rates elicited by the three different juices. We did this for each neuron that showed selectivity in any of the four epochs, once again focusing on the epoch that showed maximal selectivity. In subject $G$, all six possible rankings of the three juices were equally prevalent and none occurred any more frequently than would be expected by chance (expected frequency $=0.17$, binomial test, $p>0.1$ in all cases). In subject $\mathrm{C}$, there was a slight tendency for neurons to show their lowest firing rate to orange and their highest firing rate to guava (18 of 69 or $26 \%$, binomial test, $p<0.05$ ). To examine whether this related to the subject's preferences among the juices, we used a choice paradigm that we have previously used to study decision-making in monkeys (Kennerley et al., 2008). Briefly, we presented the subject with two pictures from a set of three. The subject selected one of the pictures by making a lever movement and received the reward associated with that picture. Each of the three rewards was associated with a specific picture. Subject $C$ chose orange juice over guava on 25 of 32 trials, guava over tomato on 18 of 26 trials and orange juice over tomato on 19 of 27 trials. Each of these proportions was statistically significant (binomial test, $p<0.05$ in all three cases). This revealed that the subject preferred orange juice over guava and guava over tomato. Thus, there did not appear to be a relationship between subject C's preferences and the most common rank ordering of the juices by the neurons. In summary, there was little evidence to suggest that neurons favored a particular encoding of the juices, or that subjective preferences mapped onto neuronal preferences in this particular task. Although we did not break the analysis down by brain area (because there would have been too few neurons encoding any specific rank order) there did not appear to be any encoding of rank order in any of the areas.

\section{Match/nonmatch selectivity}

To characterize neuronal selectivity during the juice 2 and preresponse epochs, we used a two-way ANOVA with factors of juice (the identity of the second juice) and response (whether the juice was a match or nonmatch and consequently whether the subject
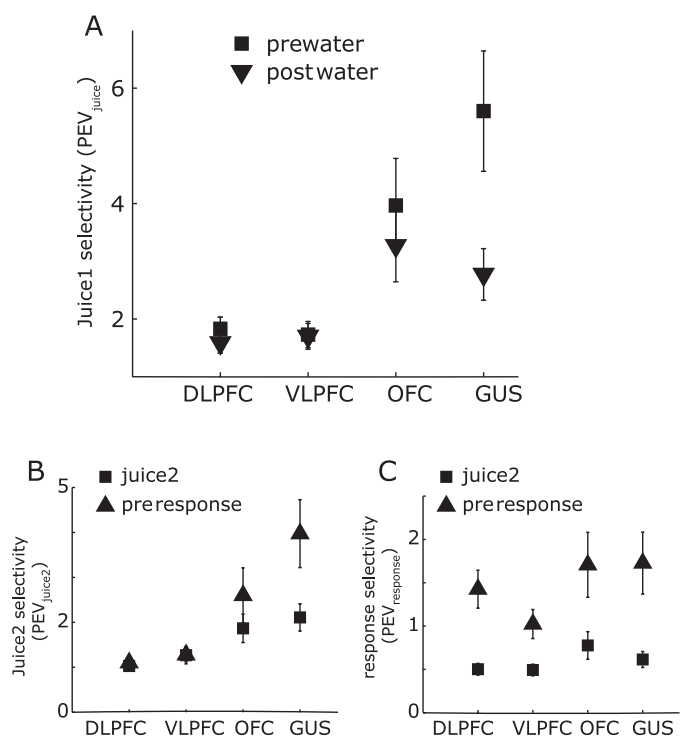

Figure 4. $\quad \boldsymbol{A}$, The mean strength of neuronal selectivity for the encoding of the gustatory stimulus ( $\mathrm{PEV}_{\text {juice }}$ ) during the pre- and postwater epochs. There was stronger encoding in OFC and GUS relative to DLPFC and VLPFC. In addition, OFC neurons encoded the juice in WM with approximately the same strength in the pre- and postwater epochs whereas GUS neurons showed weaker selectivity during the postwater epoch compared with the prewater epoch. $\boldsymbol{B}$, The mean strength of neuronal selectivity for the encoding of the gustatory stimulus ( $\mathrm{PEV}_{\text {juice }}$ ) during the juice 2 and preresponse epochs. Selectivity was stronger during the preresponse epochs than the juice 2 epochs and strongest in GUS than the other brain areas. $\boldsymbol{C}$, The mean strength of neuronal selectivity for the encoding of the behavioral response $\left(P E V_{\text {response }}\right)$ during the juice 2 and preresponse epochs. Response selectivity was significantly higher during the preresponse epoch compared with the juice 2 epoch.

had to release or hold the lever respectively). During both the juice 2 and the preresponse epochs, only 9 of 392 (2\%) of the neurons showed a significant interaction between the two factors. Given the small proportion of such neurons, we did not analyze them further. Instead, we focused on the majority of the selective neurons that showed a main effect of juice and/or response.

During the juice 2 epoch, the majority of the selective neurons showed a significant main effect of juice (Table 2). However, there were no significant differences in the proportion of selective neurons between the areas (all comparisons, $\chi^{2}<2.7, p>0.05$ ). The number of neurons that showed a main effect of response during the juice 2 epoch did not exceed that expected by chance in any area (binomial, $p>0.1$ ). Thus, neuronal selectivity during the juice 2 epoch appeared to relate primarily to the identification of the presented juice.

During the preresponse epoch, neurons showing a main effect of juice were only present in OFC and GUS. The proportion in DLPFC or VLPFC did not exceed that expected by chance (binomial, $p>0.1$ ). There were more juice-selective neurons in GUS than OFC and the difference in the proportions approached significance $\left(\chi^{2}=3.4, p=0.06\right)$. Many neurons also showed a 
Table 2. Percentage of neurons that encoded the identity of the second juice or the upcoming behavioral response during the juice 2 and preresponse epochs

\begin{tabular}{llllll}
\hline & \multicolumn{2}{l}{ Response } & & Juice & \\
\cline { 2 - 3 } \cline { 5 - 5 } & Juice2 & Preresponse & & Juice2 & Preresponse \\
\hline DLPFC & $1^{\text {ns }}$ & 16 & $1^{\text {ns }}$ & $1^{\text {ns }}$ \\
VLPFC & $3^{\text {ns }}$ & 11 & 6 & $3^{\text {ns }}$ \\
OFC & $0^{\text {ns }}$ & 5 & 8 & 9 \\
GUS & $2^{\text {ns }}$ & 9 & 14 & 21 \\
\hline
\end{tabular}

ns indicates that the proportion of selective neurons did not exceed that expected by chance (binomial test, $p>0.1$ ).

Table 3. Percentage of neurons showing task-related activity, defined as significant change in firing rate from the $1000 \mathrm{~ms}$ baseline period

\begin{tabular}{lllllll}
\hline & Prejuice & Juice1 & Prewater & Postwater & Juice2 & Response \\
\hline DLPFC & 35 & 42 & 49 & 44 & 30 & 36 \\
VLPFC & 35 & 41 & 50 & 47 & 34 & 39 \\
OFC & $\mathbf{5 8}$ & 43 & $\mathbf{4 8}$ & 47 & 38 & $\mathbf{4 7}$ \\
GUS & $\mathbf{5 6}$ & 52 & $\mathbf{7 6}$ & $\mathbf{7 9}$ & 32 & $\mathbf{5 6}$ \\
\hline
\end{tabular}

Within each task epoch, bold indicates proportions that are significantly different from others in the same column ( $\chi^{2}$ test, $p<0.05$ ). factors. There was a significant main effect of epoch $\left(F_{(1,775)}=34, p<1 \times 10^{-8}\right)$, but no significant main effect of area $\left(F_{(3,775)}\right.$ $=1.9, p>0.1)$ nor a significant area $X$ epoch interaction $\left(F_{(3,775)}<1, p>0.1\right)$.

We determined the latency at which neurons encoded the behavioral response using a sliding ANOVA analysis. We defined the latency of selectivity as the time when the $p$-value fell below 0.005 for three consecutive time bins (see Materials and Methods). A similar proportion of neurons reached this criterion in all four new areas (DLPFC, 37 of 141 or $26 \%$; VLPFC, 23 of 89 or $26 \%$; OFC, 24 of 76 or $32 \%$; and GUS, 30 of 86 or $35 \%$ ). There was no significant difference between the areas with regard to the time at which they first encoded the behavioral response (DLPFC, $686 \pm 38 \mathrm{~ms}$; VLPFC, $665 \pm 65 \mathrm{~ms}$; OFC, significant main effect of response. However, the only significant difference was a greater proportion of response-selective neurons in DLPFC relative to OFC $\left(\chi^{2}=4.6, p<0.05\right)$; all other comparisons between areas were not significant $\left(\chi^{2}<1.7, p>0.05\right)$. There was a similar number of response-selective neurons that showed a higher firing rate when the subject had to release the lever (59\%) as when the subject held the lever (41\%). These proportions did not significantly differ from an even split (binomial test, $p>0.1$ ). Note that there are a number of potential explanations for response-selective neurons. In addition to the behavioral response, they might also reflect the match/nonmatch decision, or even a reward expectancy signal, because the subject will receive the final reward for correct performance slightly earlier on match trials (in which they immediately release the lever) compared with nonmatch trials (in which they must hold the lever for $900 \mathrm{~ms})$.

As well as determining the proportion of selective neurons, we also quantified the strength of encoding by calculating the PEV because of both experimental factors $\left(\mathrm{PEV}_{\text {juice }}\right.$ and $\left.\mathrm{PEV}_{\text {response }}\right)$ during the juice2 and preresponse epochs (see Materials and Methods). Figure $4 B$ shows the strength of encoding of the juice $\left(\mathrm{PEV}_{\text {juice }}\right)$ for each of the four brain areas and two time epochs. It was stronger during the preresponse epoch than the juice 2 epoch and strongest in GUS than the other brain areas. To analyze these effects statistically, we performed a two-way ANOVA on the $\mathrm{PEV}_{\text {juice }}$ values of the neurons with area (the four different brain areas) and epoch (juice 2 and preresponse) as factors. There was a significant area $\times$ epoch interaction $\left(F_{(3,775)}=3.2, p<0.05\right)$ which we characterized by analyzing the simple effects and using post hoc Tukey-Kramer tests (evaluated at $p<0.05$ ). There was a significant simple effect of area during the preresponse epoch $\left(F_{(3,776)}=16, p<1 \times 10^{-9}\right)$ but not the juice 2 epoch $\left(F_{(3,776)}=\right.$ $2.44, p>0.05)$. The effect during the preresponse epoch was the result of a significantly larger $\mathrm{PEV}_{\text {juice }}$ in GUS compared with DLPFC and VLPFC. No other comparisons between areas were significantly different. There was also a significant simple effect of epoch in GUS $\left(F_{(1,776)}=14, p<0.005\right)$, but not the other three areas (all tests, $F_{(1,776)}<2, p>0.1$ ).

Figure $4 C$ shows that encoding of the behavioral response $\left(\mathrm{PEV}_{\text {response }}\right)$ was significantly stronger during the preresponse epoch compared with the juice 2 epoch. We performed a two-way ANOVA on the PEV $\mathrm{P}_{\text {response }}$ values of the neurons with area (the four different brain areas) and epoch (juice 2 and preresponse) as
$624 \pm 62 \mathrm{~ms}$; GUS, $616 \pm 52 \mathrm{~ms}$; one-way ANOVA, $F_{(3,110)}<1$ $p>0.1)$.

In summary, after the presentation of the second juice, GUS neurons largely encoded its identity with neurons in PFC showing little selectivity. In contrast, neurons in all areas encoded the behavioral response. Thus, whether PFC neurons encode the juice's identity depends on the stage of the task. During the first delay (the pre- and postwater epochs), when remembering the juice's identity is essential for correct performance of the task, many PFC neurons (and particularly those in OFC) encode this information across the delay. However, by the second delay (preresponse epoch) our subjects can decide whether or not the two juices match such that they simply have to remember whether or not they intend to hold or release the lever. Indeed, this is the information encoded by PFC neurons (particularly those in DLPFC) during the second delay. This contrasts with neuronal activity in GUS, which encodes the identity of the juice regardless of the stage of the task.

\section{Task-related activity}

We quantified the prevalence of task-related activity by comparing each neuron's overall firing rate during each task epoch with its activity during the baseline epoch. The vast majority of neurons $(85 \%)$ showed a significant change in firing rate in at least one task epoch (Table 3). Of these task-related responses, there was an approximately even split between those neurons increasing their firing rate above baseline (51\%) and those neurons decreasing their firing rate below baseline (49\%). Both OFC and GUS neurons tended to increase their firing rate during the prejuicel epoch, which may reflect the anticipation of the delivery of the first juice. This type of activity was less prevalent in DLPFC and VLPFC. In addition, many GUS neurons showed an elevated firing rate during the pre- and postwater epochs, although this activity did not necessarily discriminate between the different juices. Such activity may relate to processes common to the ingestion of all three juices, or to the water drop, or reflect anticipation of upcoming juices and rewards.

Neuronal responses to juice stimuli outside of the behavioral task Neuronal responses to the juices delivered on free-juice trials enabled us to examine how quickly the different brain areas identified the juices, because on these trials there was no visual cue to alert the subject as to which juice would occur. During the juice epoch, only a small number of neurons encoded the identity of 
the juice (Table 1) and there was no significant difference between the areas in terms of the proportions of juice-selective neurons (all comparisons, $\chi^{2}<3.1, p>0.05$ ). During the postjuice epoch, there were significantly more juice-selective neurons in GUS compared with both DLPFC and VLPFC (both comparisons, $\left.\chi^{2}>10, p<0.005\right)$, and significantly more in OFC compared with DLPFC $\left(\chi^{2}=5.3, p<0.05\right)$. The remaining comparisons among areas were not significant (all comparisons, $\chi^{2}<2.2, p>0.05$ ). We compared the proportion of selective neurons during the postjuice epoch of the free reward trials with the prewater epoch in the behavioral task. We found that there was no significant difference between the number of neurons that showed juice selectivity in either epoch in any of the areas (all comparisons $\chi^{2}<1, p>0.1$ ). One potential explanation for this is that although we do not require our subjects to hold the juice in working memory on free-juice trials, this does not mean that they are not doing so. It may be easier for our subjects to hold the juice in working memory on all trials, rather than trying to not hold the juice in working memory on the small proportion of the trials that do not require it.

We determined the latency at which neurons encoded the juices' identity using a sliding ANOVA analysis. We defined the latency of selectivity as the time when the $p$-value fell below 0.005 for three consecutive time bins (see Materials and Methods). The proportion of neurons that reached this criterion in DLPFC ( 5 of 141 or $3.3 \%$ ) did not exceed that expected by chance (binomial test, $p>0.1$ ) and so we excluded them from the remainder of the analysis. In the remaining areas, 9 of $89(10 \%)$ of VLPFC neurons reached criterion, 12 of $76(16 \%)$ of OFC neurons, and 16 of 86 (19\%) of GUS neurons. There was no significant difference between the areas with regard to the time at which they first encoded the juice (VLPFC, $461 \pm 81 \mathrm{~ms}$; OFC, $511 \pm 60 \mathrm{~ms}$; GUS, $516 \pm$ $70 \mathrm{~ms}$; one-way ANOVA, $\left.F_{(2,30)}<1, p>0.1\right)$.

\section{Discussion}

Many neurons in PFC showed differential firing rates during the delay of a gustatory matching-to-sample task dependent on the identity of the first juice stimulus, consistent with a role for maintaining gustatory information in working memory. Our findings are consistent with the idea that PFC is to some extent organized according to the content of the information that must be maintained across a delay, because the strongest encoding of gustatory working memory occurred in OFC rather than VLPFC or DLPFC. This shows that OFC, an area not typically associated with working memory, can show stronger involvement in working memory than LPFC when the type of information that must be maintained relates to the type of information for which OFC is specialized to process. Indeed, this is the first time that neurons in an area outside of LPFC have shown stronger working memory selectivity than LPFC neurons themselves.

Neuroimaging studies in humans that have sought to test the content model have produced mixed results, with some studies dissociating DLPFC and VLPFC based on object and spatial working memory (Courtney et al., 1996), whereas other studies have found little evidence for the content model of prefrontal organization (Postle and D'Esposito, 1999; Postle et al., 2000). However, a strict test of the content model in humans may prove difficult. Demonstrating analogous effects in humans to the ones we saw in our task may be impossible, because one cannot eliminate the possibility that human subjects encode the gustatory stimulus with a verbal label. However, a recent neuroimaging study did conclude that OFC was particularly important for maintaining emotional information in working memory. Stern and colleagues used a matching-to-sample task with face stimuli (LoPresti et al., 2008). Subjects were required to make match judgments regarding either the identity or the emotional expression of the face. Within posterior sensory cortex, separate areas encoded these two aspects of the facial stimulus. In contrast, OFC exhibited sustained delay activity for both emotion and identity trials. This suggests an important role for OFC in holding socially and emotionally relevant information in working memory, consistent with its stronger limbic connections relative to other PFC areas.

However, we must be careful in proposing too strong a content model of PFC functional organization. OFC and LPFC differ not just in the regions with which they connect, but also in terms of their intrinsic anatomical organization (Zald, 2007). For example, LPFC has a well differentiated layer IV, which is absent in OFC (Barbas and Pandya, 1989; Carmichael and Price, 1994; Petrides and Pandya, 1994). Such anatomical differences highlight the fact that it is unlikely that all PFC areas are performing the same process, merely differing in the type of information processed depending on their anatomical connections (Zald, 2007). Instead, such differences suggest that different PFC areas perform fundamentally different computations. Our results help to constrain how those computations might differ, because it is clear that OFC neurons are capable of maintaining information across a delay consistent with a role in working memory. However, the exact functional organization of PFC remains unclear. Perhaps the most striking functional difference between OFC and LPFC, from both neuropsychological and neurophysiological studies, relates to their involvement in decision-making and goaldirected behavior (Fellows and Farah, 2005; Murray et al., 2007; Wallis, 2007). For example, in a task that involves a simple choice between two stimuli associated with different behavioral outcomes, OFC shows robust encoding of multiple variables necessary to make a decision, which is noticeably absent in LPFC (Kennerley et al., 2008).

\section{Delay activity in sensory cortex}

In addition to OFC, we also saw neuronal activity in GUS related to encoding of the identity of the juice stimulus across the delay. However, this activity differed from that in OFC in a number of key respects. First, it was noticeably weaker after the intervening distracting water drop. Miller and colleagues observed a similar effect in the visual system. Neuronal activity related to the encoding of a visual stimulus across a delay occurs in both LPFC and inferior temporal cortex. However, unlike LPFC, intervening, distracting visual stimuli disrupted the delay activity in inferior temporal cortex (Miller et al., 1996). Neuronal activity in the entorhinal cortex during visual working memory tasks is also resistant to intervening distraction (Suzuki et al., 1997). From these results, Miller concluded that the ability to maintain information across distraction was an important property of LPFC that differentiated this region from posterior sensory areas (Miller and Cohen, 2001). Our results are consistent with this assertion, because they show that neuronal activity in OFC is also more resistant to distraction than the upstream sensory area.

Second, OFC delay activity differs from GUS delay activity in that its prevalence depends on the stage of the task. There was little difference between OFC and GUS in the prevalence and strength of encoding of the identity of the first juice during the pre- and postwater epochs. In contrast, OFC encoding of the second juice during the preresponse epoch was noticeably weaker than in GUS. Thus, the encoding of gustatory information in OFC appears to depend on its relevance to the successful perfor- 
mance of the task. During the first delay, when the subject must remember the identity of the first juice, we see robust encoding of gustatory information in OFC. However, during the second delay the subject has had the opportunity to compare the first juice with the second and decide whether they match. Thus, he has simply to remember his intended behavioral response and so at this stage of the task gustatory information is irrelevant. The tendency to encode information only when it is behaviorally relevant is a hallmark feature of working memory that differentiates it from delay activity in posterior sensory cortex (Rao et al., 1997; Rainer et al., 1998; Duncan, 2001; Everling et al., 2002). It provides further support to our conclusion that gustatory encoding in OFC is not simply a continuation of the taste response to the juice, but rather reflects the active maintenance of the information in working memory.

\section{Interpretational issues}

There did not appear to be any consistent pattern in the order in which the neurons encoded the three different juices. Thus, there was no evidence that the neurons encoded the juices by ranking them in a manner consistent with the subject's preferences. This might appear to contradict recent studies suggesting that OFC plays an important role in encoding subjective preferences (Padoa-Schioppa and Assad, 2006, 2008; Fellows and Farah, 2007). However, our task did not require the subject to make judgments or choices on the basis of their preferences between the juices, but rather attend to the sensory characteristics and identity of the juices. Thus, one potential explanation for why we did not see encoding of preferences may be that OFC neurons, like neurons in other PFC areas (Rao et al., 1997; Duncan, 2001; Miller and Cohen, 2001) can alter the aspect of the stimulus that they encode in response to the overarching task demands. Indeed, recent neuroimaging findings in humans show that activity in OFC is modulated by selectively attending to either the pleasantness or intensity of a gustatory stimulus (Grabenhorst and Rolls, 2008). Future experiments could tackle this issue more directly. For example, experiments by Platt and colleagues showed that monkeys exhibit strong preferences in evaluating pictures of other monkeys (Deaner et al., 2005). If OFC neurons are encoding preferences in working memory, rather than taste information per se, one might expect such encoding to be supramodal, such that single neurons might encode both taste preferences and social preferences in working memory. Nonetheless, regardless of whether the neurons are encoding gustatory information or preference information in working memory, it is clear that if one uses the appropriate stimuli, one can get stronger working memory activity in OFC compared with LPFC.

\section{Conclusion}

In conclusion, our results demonstrate that OFC neurons can show greater encoding of information in working memory than LPFC neurons when the information consists of that for which OFC is specialized. This supports the content model regarding the functional organization of PFC, but it is unlikely to be the whole story. For example, we have previously found that OFC neurons can maintain abstract information in working memory (Wallis et al., 2001) and PFC neurons show the capacity to alter the nature of the information they are maintaining in working memory depending on the demands of the task (Rao et al., 1997). Our results do not necessarily contradict other models of working memory organization, such as that posited by Petrides, whereby VLPFC maintains information in working memory whereas DLPFC is responsible for monitoring and manipulating that information (Petrides, 1996). Indeed, a hybrid version of the two models may be the most accurate, with DLPFC manipulating and monitoring information, whereas VLPFC and OFC share the load of maintaining information dependent on informational content. Olfactory, gustatory, emotional, social and reward information may be maintained in working memory by OFC neurons, whereas somatosensory, auditory and visual information may be maintained by VLPFC neurons.

\section{References}

Barbas H (1993) Organization of cortical afferent input to orbitofrontal areas in the rhesus monkey. Neuroscience 56:841-864.

Barbas H, Pandya DN (1989) Architecture and intrinsic connections of the prefrontal cortex in the rhesus monkey. J Comp Neurol 286:353-375.

Carmichael ST, Price JL (1994) Architectonic subdivision of the orbital and medial prefrontal cortex in the macaque monkey. J Comp Neurol 346:366-402.

Carmichael ST, Price JL (1995) Sensory and premotor connections of the orbital and medial prefrontal cortex of macaque monkeys. J Comp Neurol 363:642-664.

Cavada C, Goldman-Rakic PS (1989) Posterior parietal cortex in rhesus monkey: II. Evidence for segregated corticocortical networks linking sensory and limbic areas with the frontal lobe. J Comp Neurol 287:422-445.

Courtney SM, Ungerleider LG, Keil K, Haxby JV (1996) Object and spatial visual working memory activate separate neural systems in human cortex. Cereb Cortex 6:39-49.

Deaner RO, Khera AV, Platt ML (2005) Monkeys pay per view: adaptive valuation of social images by rhesus macaques. Curr Biol 15:543-548.

Duncan J (2001) An adaptive coding model of neural function in prefrontal cortex. Nat Rev Neurosci 2:820-829.

Everling S, Tinsley CJ, Gaffan D, Duncan J (2002) Filtering of neural signals by focused attention in the monkey prefrontal cortex. Nat Neurosci 5:671-676.

Fellows LK, Farah MJ (2005) Different underlying impairments in decisionmaking following ventromedial and dorsolateral frontal lobe damage in humans. Cereb Cortex 15:58-63.

Fellows LK, Farah MJ (2007) The role of ventromedial prefrontal cortex in decision making: Judgment under uncertainty or judgment per se? Cereb Cortex 17:2669-2674.

Funahashi S, Bruce CJ, Goldman-Rakic PS (1989) Mnemonic coding of visual space in the monkey's dorsolateral prefrontal cortex. J Neurophysiol 61:331-349.

Funahashi S, Bruce CJ, Goldman-Rakic PS (1993) Dorsolateral prefrontal lesions and oculomotor delayed-response performance: evidence for mnemonic "scotomas". J Neurosci 13:1479-1497.

Goldman-Rakic PS (1987) Circuitry of primate prefrontal cortex and regulation of behavior by representational memory. In: Handbook of physiology, the nervous system, higher functions of the brain (Plum F, ed), pp 373-417. Bethesda, MD: American Physiological Society.

Goldman-Rakic PS (1996) Regional and cellular fractionation of working memory. Proc Natl Acad Sci U S A 93:13473-13480.

Grabenhorst F, Rolls ET (2008) Selective attention to affective value alters how the brain processes taste stimuli. Eur J Neurosci 27:723-729.

Hikosaka K, Watanabe M (2000) Delay activity of orbital and lateral prefrontal neurons of the monkey varying with different rewards. Cereb Cortex 10:263-271.

Kennerley SW, Dahmubed AF, Lara AH, Wallis JD (2008) Neurons in the frontal lobe encode the value of multiple decision vairables. J Cogn Neurosci. Advance online publication. Retrieved September 25, 2008. doi:10.1162/jocn.2009.21100.

LoPresti ML, Schon K, Tricarico MD, Swisher JD, Celone KA, Stern CE (2008) Working memory for social cues recruits orbitofrontal cortex and amygdala: a functional magnetic resonance imaging study of delayed matching to sample for emotional expressions. J Neurosci 28:3718-3728.

Miller EK, Cohen JD (2001) An integrative theory of prefrontal cortex function. Annu Rev Neurosci 24:167-202.

Miller EK, Erickson CA, Desimone R (1996) Neural mechanisms of visual working memory in prefrontal cortex of the macaque. J Neurosci 16:5154-5167.

Mishkin M, Manning FJ (1978) Non-spatial memory after selective prefrontal lesions in monkeys. Brain Res 143:313-323. 
Morecraft RJ, Geula C, Mesulam MM (1992) Cytoarchitecture and neural afferents of orbitofrontal cortex in the brain of the monkey. J Comp Neurol 323:341-358.

Murray EA, O’Doherty JP, Schoenbaum G (2007) What we know and do not know about the functions of the orbitofrontal cortex after 20 years of cross-species studies. J Neurosci 27:8166-8169.

Padoa-Schioppa C, Assad JA (2006) Neurons in the orbitofrontal cortex encode economic value. Nature 441:223-226.

Padoa-Schioppa C, Assad JA (2008) The representation of economic value in the orbitofrontal cortex is invariant for changes of menu. Nat Neurosci 11:95-102.

Petrides M (1996) Specialized systems for the processing of mnemonic information within the primate frontal cortex. Philos Trans R Soc Lond B Biol Sci 351:1455-1461.

Petrides M, Pandya DN (1994) Comparative architectonic analysis of the human and macaque frontal cortex. In: Handbook of neuropsychology (Boller F, Grafman J, eds), pp 17-57. New York: Elsevier.

Postle BR, D’Esposito M (1999) "What"-Then-Where" in visual working memory: an event-related fMRI study. J Cogn Neurosci 11:585-597.

Postle BR, Stern CE, Rosen BR, Corkin S (2000) An fMRI investigation of cortical contributions to spatial and nonspatial visual working memory. Neuroimage 11:409-423.

Pritchard TC, Hamilton RB, Morse JR, Norgren R (1986) Projections of thalamic gustatory and lingual areas in the monkey, Macaca fascicularis. J Comp Neurol 244:213-228.

Pritchard TC, Edwards EM, Smith CA, Hilgert KG, Gavlick AM, Maryniak TD, Schwartz GJ, Scott TR (2005) Gustatory neural responses in the medial orbitofrontal cortex of the old world monkey. J Neurosci 25:6047-6056.

Rainer G, Asaad WF, Miller EK (1998) Selective representation of relevant information by neurons in the primate prefrontal cortex. Nature 393:577-579.

Rao SC, Rainer G, Miller EK (1997) Integration of what and where in the primate prefrontal cortex. Science 276:821-824.

Romanski LM, Goldman-Rakic PS (2002) An auditory domain in primate prefrontal cortex. Nat Neurosci 5:15-16.

Romanski LM, Bates JF, Goldman-Rakic PS (1999) Auditory belt and parabelt projections to the prefrontal cortex in the rhesus monkey. J Comp Neurol 403:141-157.

Romo R, Brody CD, Hernández A, Lemus L (1999) Neuronal correlates of parametric working memory in the prefrontal cortex. Nature 399:470-473.

Suzuki WA, Miller EK, Desimone R (1997) Object and place memory in the macaque entorhinal cortex. J Neurophysiol 78:1062-1081.

Toth LJ, Assad JA (2002) Dynamic coding of behaviourally relevant stimuli in parietal cortex. Nature 415:165-168.

Tremblay L, Schultz W (1999) Relative reward preference in primate orbitofrontal cortex. Nature 398:704-708.

Wallis JD (2007) Orbitofrontal cortex and its contribution to decisionmaking. Annu Rev Neurosci 30:31-56.

Wallis JD, Miller EK (2003) From rule to response: neuronal processes in the premotor and prefrontal cortex. J Neurophysiol 90:1790-1806.

Wallis JD, Anderson KC, Miller EK (2001) Single neurons in prefrontal cortex encode abstract rules. Nature 411:953-956.

Watanabe M (1996) Reward expectancy in primate prefrontal neurons. Nature 382:629-632.

Wilson FA, Scalaidhe SP, Goldman-Rakic PS (1993) Dissociation of object and spatial processing domains in primate prefrontal cortex. Science 260:1955-1958.

Zald DH (2007) Orbital versus dorsolateral prefrontal cortex: anatomical insights into content versus process differentiation models of the prefrontal cortex. Ann N Y Acad Sci 1121:395-406. 